\title{
Determination of molecular species of calcium salts of MDP produced through decalcification of enamel and dentin by MDP-based one-step adhesive
}

\author{
Yoko YOKOTA ${ }^{1,2}$ and Norihiro NISHIYAMA ${ }^{3}$ \\ ${ }^{1}$ Nihon University Graduate School of Dentistry at Matsudo, 870-1 Sakaecho, Nishi 2, Matsudo, Chiba 271-8587, Japan \\ ${ }^{2}$ Department of Renascent Dentistry, Nihon University School of Dentistry at Matsudo, 870-1 Sakaecho, Nishi 2, Matsudo, Chiba 271-8587, Japan \\ ${ }^{3}$ Department of Dental Biomaterials, Nihon University School of Dentistry at Matsudo, 870-1 Sakaecho, Nishi 2, Matsudo, Chiba 271-8587, Japan \\ Corresponding author, Norihiro NISHIYAMA; E-mail: nishiyama.norihiro@nihon-u.ac.jp
}

\begin{abstract}
Enamel and dentin particles were added to an experimental 10-methacryloyloxydecyl dihydrogen phosphate (MDP)-based one-step adhesive to react for $30 \mathrm{~s}$. After enamel and dentin reactants were analyzed using X-ray diffraction (XRD) and phosphorus-31 nuclear magnetic resonance $\left({ }^{31} \mathrm{P} \mathrm{NMR}\right)$ techniques, curve-fitting analysis was performed on the ${ }^{31} \mathrm{P}$ NMR spectra of enamel and dentin reactants. By varying the molar ratio of calcium chloride to MDP, a series of three types of MDP-Ca salts were synthesized. The molecular species of calcium salts of MDP (MDP-Ca salts) produced by decalcification of enamel or dentin were determined based on the XRD and ${ }^{31} \mathrm{P}$ NMR analysis results of these three types of synthesized MDP-Ca salts. Curve-fitting analysis showed that enamel and dentin developed several types of MDP-Ca salts and amorphous dicalcium phosphate dihydrate (DCPD) during decalcification. The molecular species of MDP-Ca salts produced by enamel and dentin were mono-calcium salts of MDP monomer and MDP dimer. In addition, dentin produced a di-calcium of MDP dimer.
\end{abstract}

Keywords: One-step adhesive, Decalcification, MDP-Ca salt, ${ }^{31} \mathrm{P}$ NMR, X-ray diffraction

\section{INTRODUCTION}

The increasingly widespread use of one-step and twostep self-etch adhesive systems over the etch-andrinse adhesive system is chiefly due to their simplified application procedures and low technique sensitivity ${ }^{1-3)}$. An acidic monomer used in these systems plays a key role in binding to enamel and dentin. The acidic monomer, such as a phosphomethacrylic monomer or carboxymethacrylic monomer, decalcifies enamel and dentin - thereby enhancing the bonding of these systems to hard tissue surfaces ${ }^{4-11}$.

To understand the adhesion mechanisms of these adhesive systems, details of the interaction between acidic monomers and tooth apatite have been investigated using X-ray photoelectron spectroscopy ${ }^{12-14)}$, $\mathrm{X}$-ray diffraction (XRD) ${ }^{15,16)}$, and phosphorus-31 nuclear magnetic resonance $\left({ }^{31} \mathrm{P} \mathrm{NMR}\right)^{17,18)}$. These reports noted that the acidic monomer, such as 10-methacryloyloxydecyl dihydrogen phosphate (MDP), produced a calcium salt of MDP (MDP-Ca salt) through the decalcification process of synthesized hydroxyapatite, enamel or dentin. Dicalcium phosphate dihydrate (DCPD) with a crystalline phase was produced at the same time when these adhesive systems were applied to synthesized hydroxyapatite or enamel ${ }^{15,17)}$.

However, the molecular species of MDP-Ca salt produced by decalcification of enamel and dentin remained to be clarified. This is important because the MDP-Ca salt produced is embedded within the created adhesive layer and may cause the latter to degrade

Color figures can be viewed in the online issue, which is available at J-STAGE.

Received Sep 22, 2014: Accepted Dec 26, 2014

doi:10.4012/dmj.2014-260 JOI JST.JSTAGE/dmj/2014-260 during the aging period. In a report by Takahashi ${ }^{19)}$, dentin developed a greater amount of MDP-Ca salt than enamel and showed a greater reduction in bond strength than enamel after thermocycling. It was also found that reduction in dentin bond strength increased with an increase in the amount of MDP-Ca salt produced, in contrast to enamel bond strength which remained unchanged.

In this study, enamel and dentin particles were added to an experimental MDP-based one-step adhesive and their reactants were analyzed using XRD and ${ }^{31} \mathrm{P}$ NMR techniques. By varying the molar ratio of calcium chloride to MDP, a series of three types of MDP$\mathrm{Ca}$ salts were synthesized. The molecular species of MDP-Ca salts produced by decalcification of enamel or dentin were then determined based on the XRD and ${ }^{31} \mathrm{P}$ NMR analysis results of these three types of synthesized MDP-Ca salts.

The null hypotheses of this study were: (1) MDPCa salt produced by decalcification of enamel or dentin was a mixture of MDP-Ca salts with different molecular species; and (2) Molecular species of MDP-Ca salts produced by enamel were the same as those by dentin.

\section{MATERIALS AND METHODS}

All chemical reagents are purchased from Wako Pure Chemical Industries (Osaka, Japan), unless otherwise indicated.

Synthesis of MDP-Ca salts

As previously described ${ }^{17}$, a series of three types of MDP-Ca salts were synthesized by varying the molar 
ratio of calcium chloride $\left(\mathrm{CaCl}_{2}\right)$ to $\mathrm{MDP}\left(\mathrm{CaCl}_{2} / \mathrm{MDP}\right)$.

Ethanol solution of $5 \mathrm{~mL}$, in which $0.0030 \mathrm{~mol}$ of MDP had been dissolved, was added dropwise to $5 \mathrm{~mL}$ of $50 \mathrm{vol} \%$ aqueous ethanol solution in which 0.0015 , 0.0030 , or $0.0060 \mathrm{~mol}$ of $\mathrm{CaCl}_{2}$ had been dissolved under stirring. After $12 \mathrm{~h}$, the reactants were centrifuged and the supernatant was discarded. Thereafter, each reactant was repeatedly rinsed with an excess amount of ethanol, and then with distilled and deionized water, to completely remove unreacted $\mathrm{MDP}$ and $\mathrm{CaCl}_{2}$ respectively. Each synthesized MDP-Ca salt was dried at $20^{\circ} \mathrm{C}$ for XRD and ${ }^{31} \mathrm{P}$ NMR analyses. A series of three types of MDP-Ca salts were synthesized and expressed as MDP-0.5 Ca salt, MDP-1 Ca salt, and MDP-2 Ca salt.

\section{Preparation of experimental one-step adhesive}

EX-15 adhesive was used in this study, ${ }^{919)}$. It consisted of a base monomer, catalysts, inhibitor, filler, acetone, and water. The base monomer was prepared by mixing $15.0 \mathrm{~g}$ of MDP, $10.0 \mathrm{~g}$ of urethane dimethacrylate (Negami Kogyo, Ishikawa, Japan), $10.0 \mathrm{~g}$ of triethylene glycol dimethacrylate (ShinNakamura Chemical Co., Wakayama, Japan), and $9.4 \mathrm{~g}$ of 4-methacryloyloxyethyl trimellitic anhydride (Sun Medical, Shiga, Japan).

The experimental one-step adhesive was then prepared by diluting mixed monomer with an aqueous acetone solution, consisting of $11.2 \mathrm{~g}$ of distilled water and $69.3 \mathrm{~g}$ of acetone. The quantity of MDP in experimental one-step adhesive was $116.1 \mathrm{mg} / \mathrm{g}$.

\section{Preparation of enamel and dentin particles}

After pulp removal, bovine crown enamel was cut using an air turbine with a diamond bur under a stream of cooling water. Cut enamel particles were obtained by decanting the cooling water collected. Enamel particles were rinsed three times with distilled and deionized water, and then dried at $20^{\circ} \mathrm{C}$.

Crown dentin particles were prepared according to the procedure mentioned above for enamel particles. Primary enamel and dentin particles used in this study were of $5-20 \mu \mathrm{m}$ in size.

Preparation of enamel and dentin reactants of experimental one-step adhesive

Bovine enamel or dentin particles $(0.200 \mathrm{~g})$ were suspended in experimental one-step adhesive (1.000 g), and the suspensions were vibrated for $30 \mathrm{~s}$ at $20^{\circ} \mathrm{C}$. After the reaction, $30 \mathrm{~mL}$ of ethanol (approximately $24 \mathrm{~g}$ ) was added to each suspension to obstruct further decalcification of enamel or dentin by MDP. This was possible because the MDP concentration in experimental one-step adhesive was reduced to $116.1 / 25 \mathrm{mg} / \mathrm{g}$ by adding ethanol. Each suspension was centrifuged for $20 \mathrm{~min}$, and the supernatant was discarded. Thereafter, the enamel and dentin reactants were repeatedly rinsed four times with $30 \mathrm{~mL}$ of ethanol. After each reactant was dried at $20^{\circ} \mathrm{C}$ for $3 \mathrm{~h}$, it was analyzed using an NMR spectrometer (EX-270, JEOL, Tokyo, Japan) and an X-ray diffractometer (RINT2000, Rigaku, Tokyo, Japan).

Enamel and dentin reactants were prepared three times in order to perform XRD and NMR observations three times.

\section{Observation of XRD patterns}

The XRD patterns of synthesized MDP-Ca salts and those of enamel and dentin reactants were recorded under these conditions: $50 \mathrm{kV}$ accelerating voltage, 300 $\mathrm{mA}$ current, $\mathrm{CuK} \alpha$ wavelength of $0.1542 \mathrm{~nm}, 1.0^{\circ} / \mathrm{min}$ scan speed, $1 / 2^{\circ}$ divergence slit, $0.73 \mathrm{~mm}$ scattering slit, $0.3 \mathrm{~mm}$ receiving slit, $0.02^{\circ}$ step width, $20.0 \mathrm{rpm}$ rotation rate, $1.6-70^{\circ}$ scanning range ( $2 \theta$ degree), and graphite crystal monochromator. The XRD patterns of powdered enamel, dentin and DCPD with a crystalline phase (Taihei Chemical Industrial Co., Ltd., Tokyo, Japan) were also measured.

Data obtained were analyzed using Jade software version 5 (Materials Data Inc., USA). Reference database was JCPDS card (Joint Committee on Powder Diffraction Standards).

\section{Observation of solid-state ${ }^{31} P$ NMR spectra}

${ }^{31} \mathrm{P}$ NMR spectra of synthesized MDP-Ca salts and those of enamel and dentin reactants were observed. Contact, repetition, and accumulation times were 2,000 $\mu \mathrm{s}, 20.05$ $\mathrm{s}$, and 120 times respectively. ${ }^{31} \mathrm{P}$ NMR chemical shifts were expressed in ppm, with $85 \%$ of $\mathrm{H}_{3} \mathrm{PO}_{4}$ as an external reference. Additionally, the ${ }^{31} \mathrm{P}$ NMR spectra of enamel, dentin and DCPD powders were also measured.

Curve-fitting analyses of the corresponding ${ }^{31} \mathrm{P}$ NMR spectra were performed using OriginPro ${ }^{\circledR}$ 9.1 Data Analysis and Graphing Software (OriginLab Corp., USA). We determined the chemical shift and intensity of each simulated peak used for the curvefitting analyses of a series of three types of synthesized MDP-Ca salts and of both enamel and dentin reactants.

\section{RESULTS}

$X R D$ and ${ }^{31} P$ NMR analyses of three types of synthesized MDP-Ca salts

Figure 1 shows the XRD patterns of a series of three types of synthesized MDP-Ca salts. As shown in Fig. 1A and Table 1, the synthesized MDP-0.5 Ca salt showed asterisk-marked three characteristic XRD peaks $\left(2 \theta=2.24,4.51\right.$, and $\left.6.76^{\circ}\right)$ and several small XRD peaks $\left(2 \theta=11.32,20.41\right.$, and $22.87^{\circ}$, etc.). Increases in the molar ratio of $\mathrm{CaCl}_{2} / \mathrm{MDP}$ led to a decrease in the intensity of the asterisk-marked three characteristic XRD peaks and allowed for the appearance of black arrow-marked three characteristic $\left(2 \theta=2.42,4.88\right.$, and $\left.7.34^{\circ}\right)$ and white arrow-marked three characteristic $(2 \theta=2.58,5.22$, and $7.86^{\circ}$ ) XRD peaks as shown in Figs. 1B and 1C.

Figure 2 shows the ${ }^{31} \mathrm{P}$ NMR spectra of three synthesized MDP-Ca salts, and the curve-fitting analysis results of the ${ }^{31} \mathrm{P}$ NMR spectra. ${ }^{31} \mathrm{P}$ NMR analysis confirmed the XRD analysis results. As shown in Fig. 2A, MDP-0.5 Ca salt developed a small NMR 


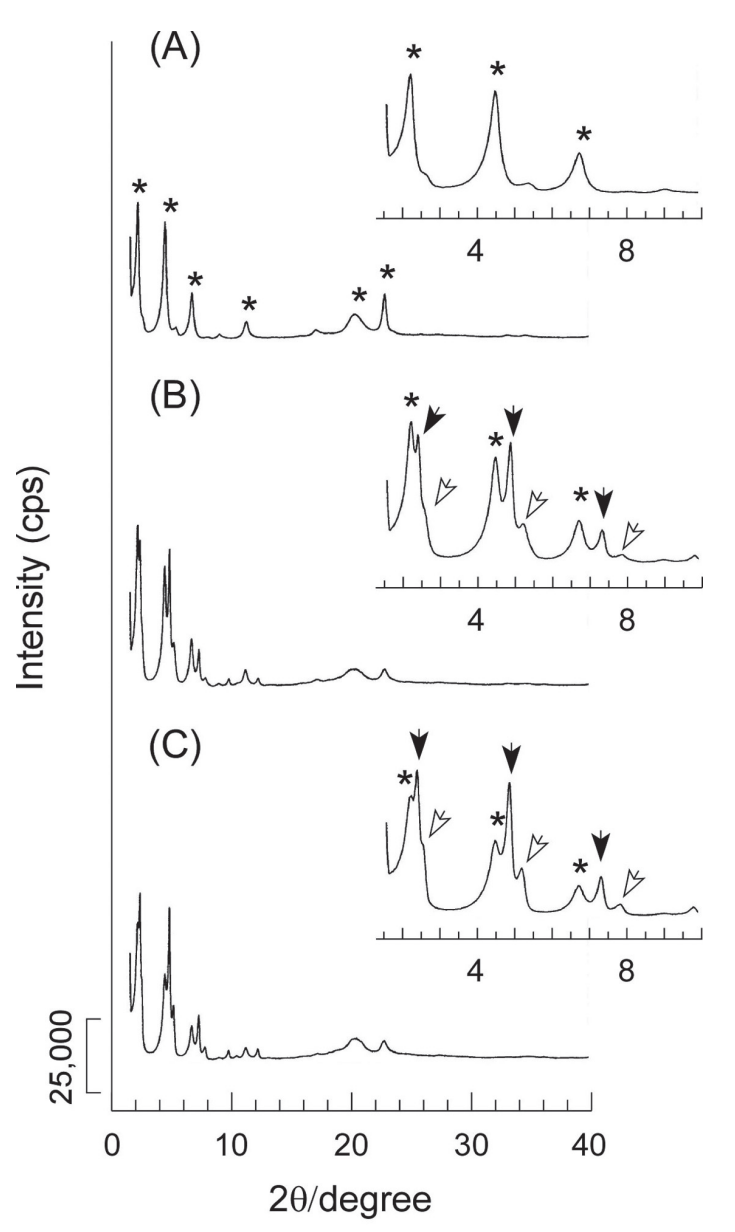

Fig. 1 XRD patterns of a series of three types of MDP$\mathrm{Ca}$ salts synthesized by varying the molar ratio of $\mathrm{CaCl}_{2} / \mathrm{MDP}$.

(A) MDP-0.5 Ca salt; (B) MDP-1 Ca salt; and (C) MDP-2 Ca salt.

peak "2" at 1.00 ppm and a broad NMR peak at 0.7$-5.1 \mathrm{ppm}$. The latter broad NMR peak had its peak at -2.13 ppm (NMR peak "6") and two shoulders at -1.41 ppm (NMR peak "5") and -3.91 ppm (NMR peak “8”). An increase in the molar ratio of $\mathrm{CaCl}_{2} / \mathrm{MDP}$ (Fig. 2B) allowed for the appearance of two NMR peaks " 1 " (1.67 ppm) and " 3 " (0.64 ppm), and NMR peak " 4 " (-0.43 ppm) which was detected at the left side of this broad peak. Due to the appearance of newly developed NMR peak "7", the broad NMR peak was shifted to a higher field by approximately 1 ppm (Fig. 2C).

However, we did not identify the existence of two NMR peaks " 2 " and " 8 " in the ${ }^{31} \mathrm{P}$ NMR spectra of both MDP-1 and MDP-2 Ca salts, since several NMR peaks were detected alongside the NMR peaks " 2 " and " 8 ".

Based on these analysis results, the ${ }^{31} \mathrm{P}$ NMR spectra of the series of three types of synthesized MDPCa salts were curve-fitted using simulated peaks "1", “2”, “3”, “4”, “5”, “6”, “7” and "8". The chemical shifts of simulated peaks used for the curve-fitting analyses

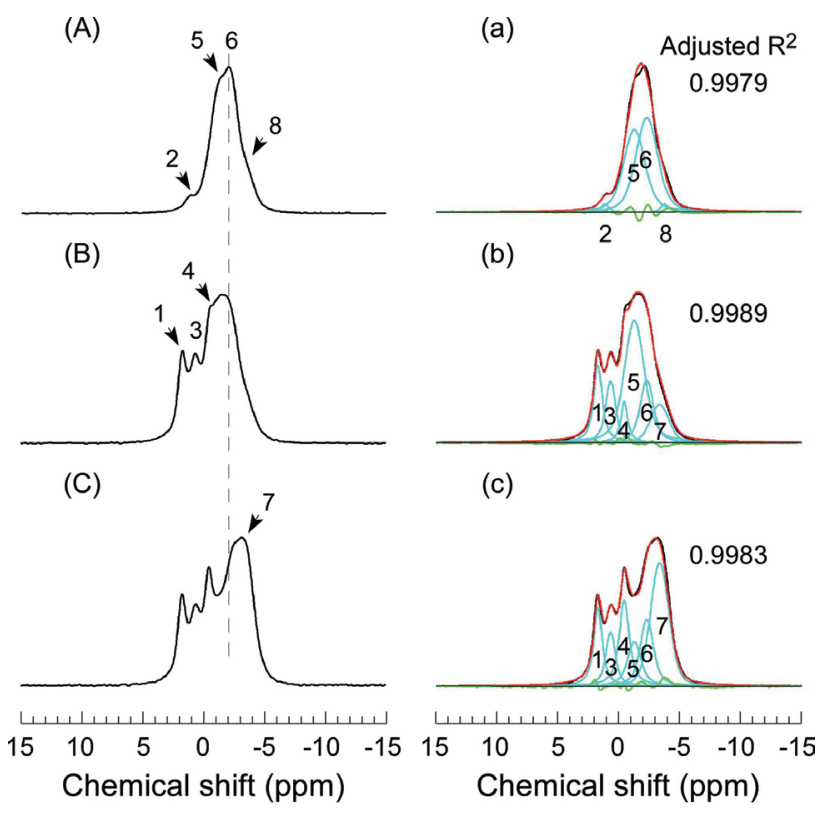

Fig. $2{ }^{31} \mathrm{P}$ NMR spectra of a series of three types of MDPCa salts synthesized by varying the molar ratio of $\mathrm{CaCl}_{2} / \mathrm{MDP}$, and the curve-fitting analysis results of the ${ }^{31} \mathrm{P}$ NMR spectra.

(A) ${ }^{31} \mathrm{P}$ NMR spectrum of MDP-0.5 Ca salt; (B) ${ }^{31} \mathrm{P}$ NMR spectrum of MDP-1 Ca salt; and (C) ${ }^{31} \mathrm{P}$ NMR spectrum of MDP-2 Ca salt.

(a) Curve-fitting analysis result of ${ }^{31} \mathrm{P} \mathrm{NMR}$ spectrum of MDP-0.5 Ca salt; (b) Curve-fitting analysis result of ${ }^{31} \mathrm{P}$ NMR spectrum of MDP-1 Ca salt; and (c) Curve-fitting analysis result of ${ }^{31} \mathrm{P}$ NMR spectrum of MDP-2 Ca salt. The black line shows the original ${ }^{31} \mathrm{P}$ NMR spectrum of each MDP-Ca salt synthesized, the sky-blue lines show the simulated peaks used for the curve-fitting of each MDP-Ca salt, the red line shows the synthetic spectrum obtained by the curve-fitting analysis of each synthesized MDP-Ca salt, and the green line is obtained after subtracting the synthetic spectrum from the original ${ }^{31} \mathrm{P}$ NMR spectrum of each synthesized MDP-Ca salt.

of three synthesized MDP-Ca salts are summarized in Table 2 .

As shown in Fig. 2a, MDP-0.5 Ca salt was successfully curve-fitted using four simulated peaks " 2 ", "5", "6" and "8". With the exception of two simulated peaks "2" and "8", MDP-1 and MDP-2 Ca salts were also curve-fitted using six simulated peaks "1", "3", “4", "5", "6"and "7" as shown in Figs. $2 \mathrm{~b}$ and $2 \mathrm{c}$ respectively.

The effect of the molar ratio of $\mathrm{CaCl}_{2} / \mathrm{MDP}$ on the intensity ratio of eight simulated peaks used for the curve-fitting analyses is illustrated in Fig. 3. An increase in the molar ratio of $\mathrm{CaCl}_{2} / \mathrm{MDP}$ allowed for the appearance of simulated peaks " 1 ", " 3 ", and " 4 "; however, it led to a drastic decrease in the intensity of simulated peak " 6 ". Further increase in the molar 
Table 1 The $2 \theta$ (degree) and $d$-spacing values ( $\AA$ ) of the diffraction peaks detected in the XRD pattern of a series of three types of MDP-Ca salt synthesized

\begin{tabular}{|c|c|c|c|c|c|}
\hline \multicolumn{2}{|c|}{ MDP-0.5 Ca salt } & \multicolumn{2}{|c|}{ MDP-1 Ca salt } & \multicolumn{2}{|c|}{ MDP-2 Ca salt } \\
\hline $2 \theta$ & d-space & $2 \theta$ & $\mathrm{d}$-space & $2 \theta$ & $\mathrm{d}$-space \\
\hline \multirow{3}{*}{2.24} & 39.42 & 2.24 & 39.41 & 2.24 & 39.43 \\
\hline & & 2.42 & 36.55 & 2.42 & 36.49 \\
\hline & & 2.61 & 33.82 & 2.58 & 34.23 \\
\hline \multirow{3}{*}{4.51} & 19.58 & 4.50 & 19.62 & 4.50 & 19.62 \\
\hline & & 4.89 & 18.04 & 4.88 & 18.09 \\
\hline & & 5.24 & 16.85 & 5.22 & 16.92 \\
\hline \multirow[t]{3}{*}{6.76} & 13.06 & 6.74 & 13.11 & 6.74 & 13.10 \\
\hline & & 7.36 & 12.00 & 7.34 & 12.03 \\
\hline & & 7.88 & 11.21 & 7.86 & 11.24 \\
\hline \multirow[t]{2}{*}{11.32} & 7.81 & 11.26 & 7.85 & 11.27 & 7.84 \\
\hline & & 12.31 & 7.18 & 12.29 & 7.20 \\
\hline 20.41 & 4.35 & 20.41 & 4.35 & 20.41 & 4.35 \\
\hline 22.87 & 3.89 & 22.87 & 3.89 & 22.87 & 3.89 \\
\hline
\end{tabular}

Table 2 The chemical shift ( $\mathrm{ppm}$ ) of the simulated peaks, their peak assignments, and molecular species of MDP-Ca salt

\begin{tabular}{|c|c|c|c|c|}
\hline Simulated peak & Chemical shift & Phosphorus atom & Molecular species & Type \\
\hline “1” & 1.695 & & $\begin{array}{c}\mathrm{O} \\
\text { II } \\
-\mathrm{CH}_{2}-\mathrm{O}-\mathrm{P}-\mathrm{O}-\mathrm{Ca}-\mathrm{OH} \\
\text { O- } \mathrm{Oa}-\mathrm{OH}\end{array}$ & IV \\
\hline “2” & 1.127 & 0 & $\begin{array}{cc}\mathrm{O} & \mathrm{O} \\
\text { II } & \text { II } \\
-\mathrm{CH}_{2}-\mathrm{O}-\mathrm{P}-\mathrm{O}-\mathrm{Ca}-\mathrm{O}-\mathrm{P}-\mathrm{O}-\mathrm{CH}_{2}- & \\
\text { O- } \mathrm{Ca}-\mathrm{OH} & \text { OH }\end{array}$ & III \\
\hline “3” & 0.655 & $\begin{array}{c}-\mathrm{CH}_{2}-\mathrm{O}-\mathrm{II}-\mathrm{O}-\mathrm{R} \\
\mathrm{I}-\mathrm{O}-\mathrm{R}\end{array}$ & $\begin{array}{c}\mathrm{O} \\
-\mathrm{CH}_{2}-\mathrm{O}-\mathrm{P}-\mathrm{O}-\mathrm{Ca}-\mathrm{O}-\mathrm{O} \\
\mathrm{O}-\mathrm{O}-\mathrm{Ca}-\mathrm{O}^{-}\end{array}$ & V \\
\hline “4” & -0.460 & & 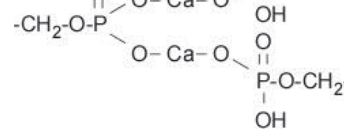 & VI \\
\hline “5” & -1.296 & & $\begin{array}{c}\mathrm{O} \\
-\mathrm{CH}_{2}-\mathrm{O}-\mathrm{P}-\mathrm{O}-\mathrm{Ca}-\mathrm{OH} \\
\text { OH }\end{array}$ & I \\
\hline "6" & -2.320 & & $\begin{array}{cc}\mathrm{O} & \mathrm{O} \\
\text { II } & \text { II } \\
\mathrm{CH}_{2}-\mathrm{O}-\mathrm{P}-\mathrm{O}-\mathrm{Ca}^{-} & \mathrm{O}-\mathrm{P}-\mathrm{O}-\mathrm{CH}_{2}- \\
\mathrm{O} & \mathrm{O}\end{array}$ & II \\
\hline "7" & -3.380 & $\begin{array}{c}\mathrm{O} \\
11 \\
-\mathrm{CH}_{2}-\mathrm{O}-\mathrm{P}-\mathrm{O}-\mathrm{R} \\
\mathrm{I} \\
\mathrm{OH}\end{array}$ & 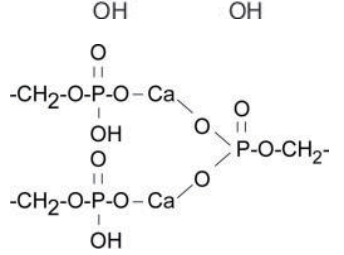 & VI \\
\hline “8” & -3.817 & & $\begin{array}{cc}\mathrm{O} & \mathrm{O} \\
\text { II } & \text { II } \\
-\mathrm{CH}_{2}-\mathrm{O}-\mathrm{P}-\mathrm{O}-\mathrm{Ca}-\mathrm{O}-\mathrm{P}-\mathrm{O}-\mathrm{CH}_{2}- \\
\mathrm{OH} & \mathrm{O}-\mathrm{Ca}-\mathrm{OH}\end{array}$ & III \\
\hline
\end{tabular}




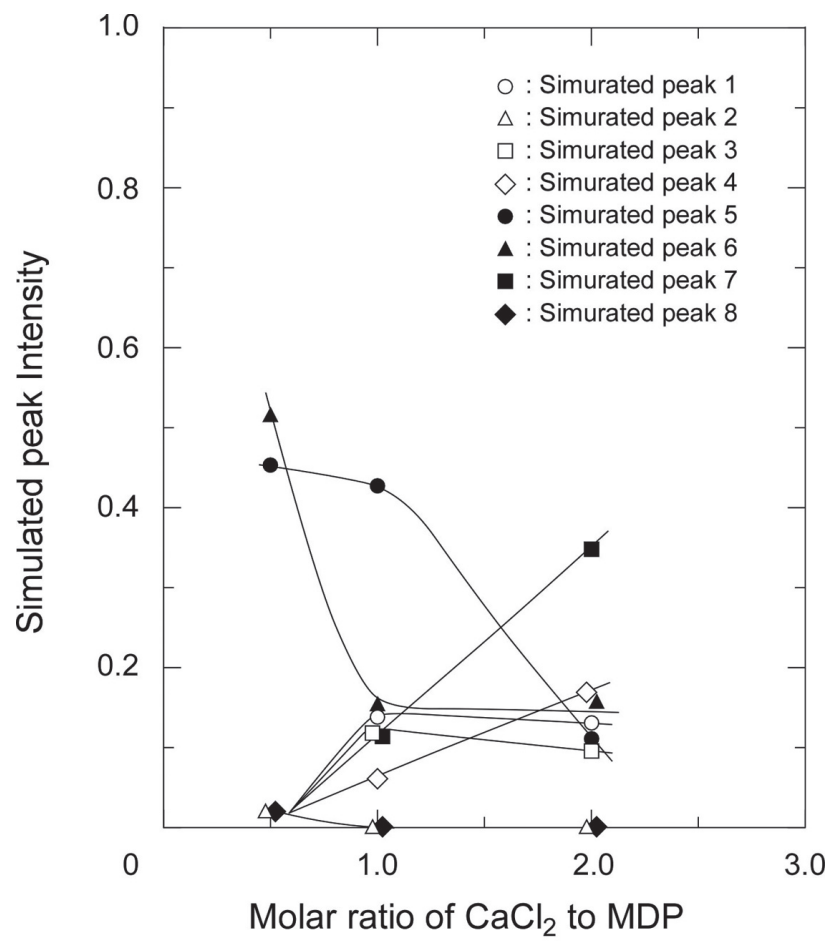

Fig. 3 Effect of the molar ratio of $\mathrm{CaCl}_{2} / \mathrm{MDP}$ on the intensity ratio of eight simulated peaks used for curve-fitting analyses.

White circles show the intensity ratio of simulated peak "1", white triangles show the intensity ratio of simulated peak " 2 ", white squares show the intensity ratio of simulated peak " 3 ", white rhombs show the intensity ratio of simulated peak " 4 ", black circles show the intensity ratio of simulated peak " 5 ", black triangles show the intensity ratio of simulated peak " 6 ", black squares show the intensity ratio of simulated peak "7", and black rhombs show the intensity ratio of simulated peak "8".

ratio resulted in further increases in the intensities of simulated peaks " 4 " and " 7 "; in contrast, it led to a decrease in the intensity of simulated peak " 5 ".

XRD and ${ }^{31} P$ NMR analyses of enamel, dentin and $D C P D$

Figure 4 shows the XRD patterns of enamel, dentin and DCPD. Figure 5 shows the ${ }^{31} \mathrm{P}$ NMR spectra of enamel, dentin and DCPD, and the curve-fitting analysis results of ${ }^{31} \mathrm{P}$ NMR spectra.

Enamel showed a typical XRD pattern of hydroxyapatite (Fig. 4A) and a sharp NMR peak assigned to the phosphorus atom of hydroxyapatite (Fig. 5A). In contrast, dentin showed a very broad XRD pattern (Fig. 4B) and NMR peak (Fig. 5B), due to the crystallinity of the dentin apatite being lower than that of enamel apatite. DCPD with a crystalline phase showed a typical XRD pattern (brushite, JCPDS 11-

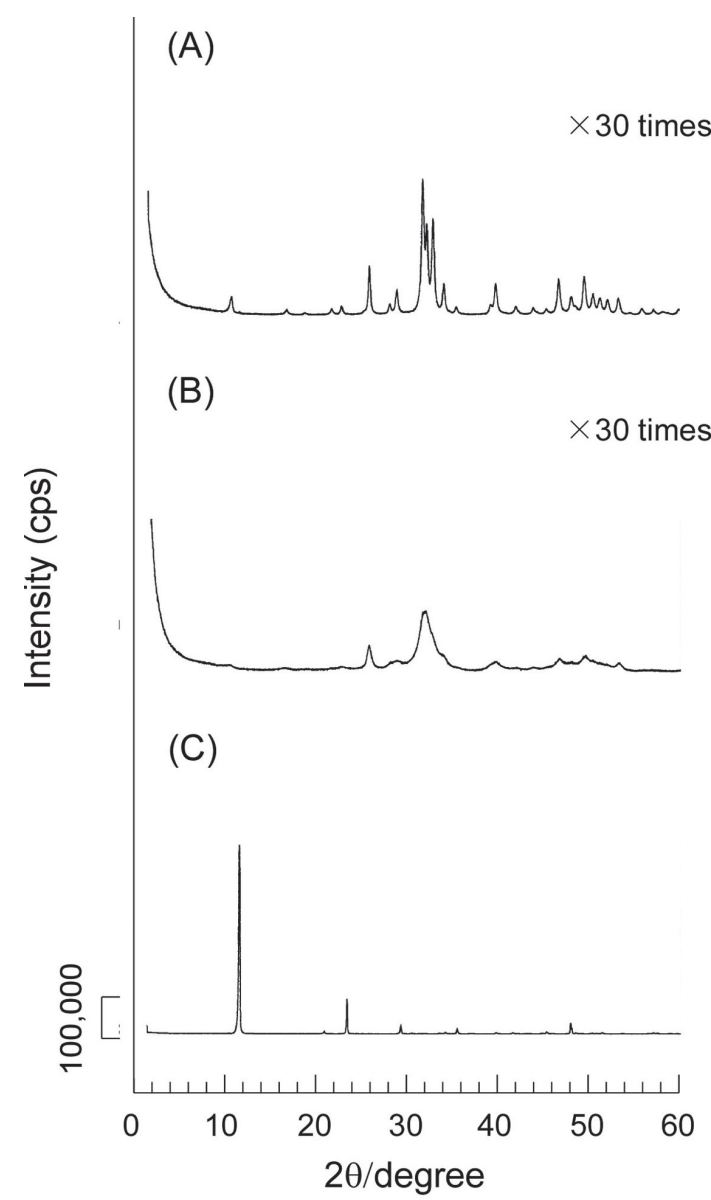

Fig. 4 XRD patterns of enamel, dentin and DCPD.

(A) Enamel; (B) Dentin; and (C) DCPD with a crystalline phase.

0293) (Fig. 4C) and a sharp NMR peak (Fig. 5C).

Based on these results, the ${ }^{31} \mathrm{P}$ NMR spectra of enamel and dentin were curve-fitted using simulated peaks " $\alpha$ " and " $\beta$ " respectively (Figs. 5a and b). The ${ }^{31} \mathrm{P}$ NMR spectrum of DCPD with a crystalline phase was curve-fitted using the simulated peak " $\gamma_{1}$ " (Fig. 5c). Chemical shifts of the simulated peaks as determined for the curve-fitting analyses of enamel, dentin, and DCPD are shown in Table 3. The half width of simulated peak " $\gamma_{1}$ " for DCPD with a crystalline phase was $95.1 \mathrm{~Hz}$.

XRD and ${ }^{31} P$ NMR analyses of enamel and dentin reactants with experimental one-step adhesive

Figure 6 shows the typical XRD patterns of enamel and dentin reactants with the experimental adhesive and that of synthesized MDP-0.5 Ca salt. Figure 7 shows the typical ${ }^{31} \mathrm{P}$ NMR spectra of enamel and dentin reactants with the experimental adhesive and that of MDP-0.5 Ca salt, and the curve-fitting analysis results of ${ }^{31} \mathrm{P}$ NMR spectra. 
(A)

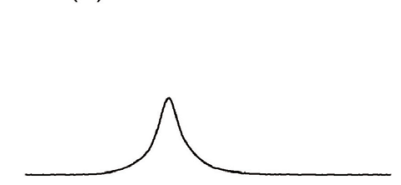

(B)

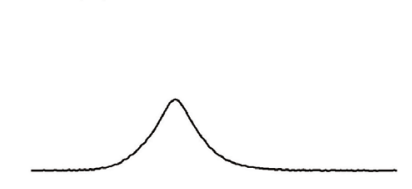

(C)

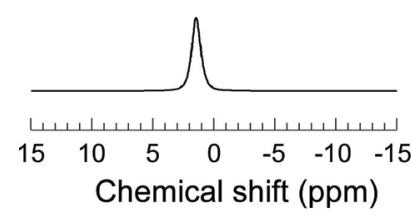

(a)

Adjusted $\mathrm{R}^{2}$

0.9948

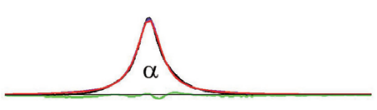

(b)

0.9925

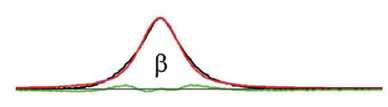

(c)

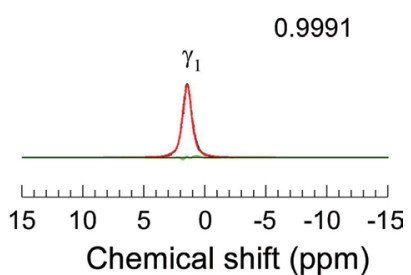

Fig. $5 \quad{ }^{31} \mathrm{P}$ NMR spectra of enamel, dentin and DCPD, and the curve-fitting analysis results of the ${ }^{31} \mathrm{P}$ NMR spectra.

(A) ${ }^{31} \mathrm{P}$ NMR spectrum of enamel; (B) ${ }^{31} \mathrm{P}$ NMR spectrum of dentin; and (C) ${ }^{31} \mathrm{P}$ NMR spectrum of DCPD with a crystalline phase.

(a) Curve-fitting analysis result of ${ }^{31} \mathrm{P}$ NMR spectrum of enamel; (b) Curve-fitting analysis result of ${ }^{31} \mathrm{P}$ NMR spectrum of dentin; and (c) Curve-fitting analysis result of ${ }^{31} \mathrm{P}$ NMR spectrum of DCPD with a crystalline phase. The black line shows the original ${ }^{31} \mathrm{P}$ NMR spectrum of enamel, dentin or DCPD, the purple line shows the simulated peak used for the curve-fitting of enamel or dentin, the pink line shows the simulated peak used for the curve-fitting of DCPD with a crystalline phase, the red line shows the synthetic spectrum obtained by the curve-fitting analysis of enamel, dentin or DCPD, and the green line is obtained after subtracting the synthetic spectrum from the original ${ }^{31} \mathrm{P}$ NMR spectrum of enamel, dentin or DCPD.

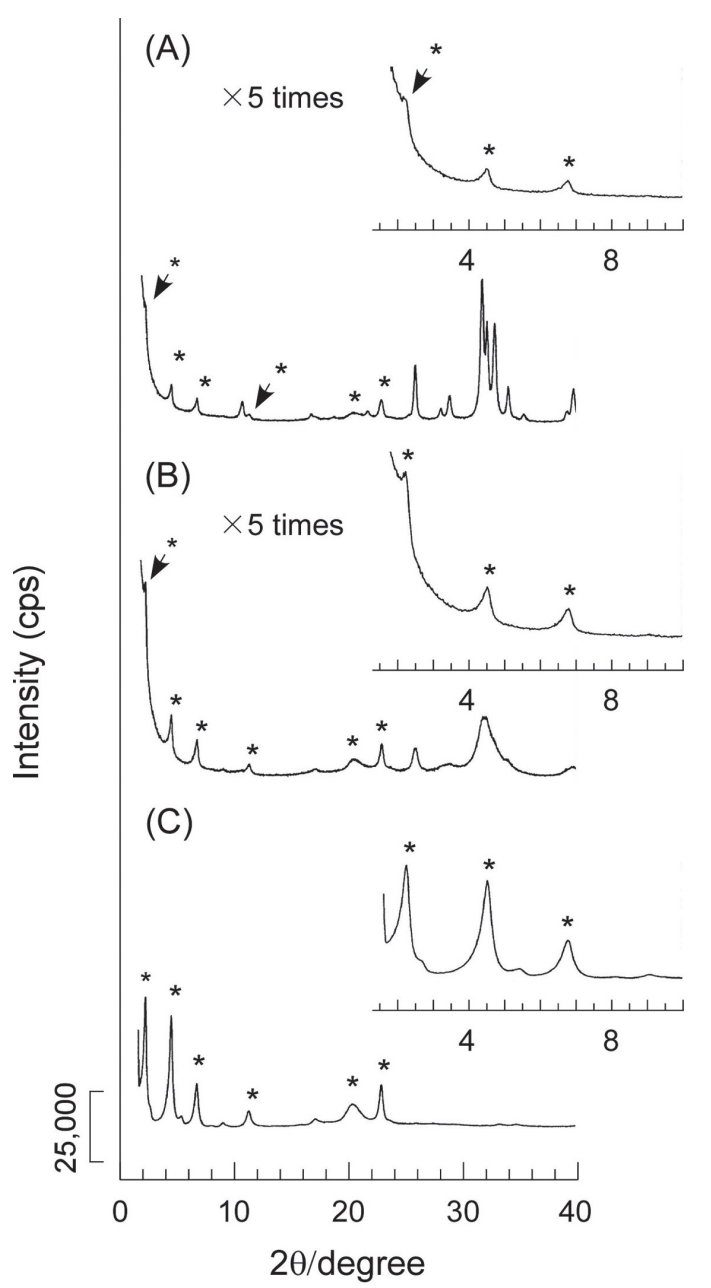

Fig. 6 XRD patterns of enamel and dentin reactants with experimental one-step adhesive and of synthesized MDP-0.5 Ca salt.

Both enamel and dentin reactants were obtained after reacting with experimental one-step adhesive for $30 \mathrm{~s}$.

(A) XRD pattern of enamel reactant; (B) XRD pattern of dentin reactant; and $(\mathrm{C}) \mathrm{XRD}$ pattern of synthesized MDP-0.5 Ca salt.

Table 3 The peak assignments of the simulated peaks for the enamel, dentin and DCPD, and their chemical shifts (ppm)

\begin{tabular}{|c|c|c|}
\hline Phosphorus atom & Simulated peak & Chemical shift \\
\hline $\mathrm{Ca}_{10}\left(\mathrm{PO}_{4}\right)_{6}(\mathrm{OH})_{2}$ for enamel & “ $\alpha ”$ & 3.180 \\
\hline $\mathrm{Ca}_{10}\left(\mathrm{PO}_{4}\right)_{6}(\mathrm{OH})_{2}$ for dentin & “ $”$ & 3.180 \\
\hline $\mathrm{CaHPO}_{4} \cdot 2 \mathrm{H}_{2} \mathrm{O}$ (Crystalline phase) & $" \gamma_{1} "$ & 1.446 \\
\hline
\end{tabular}

Enamel and dentin reactants showed asteriskmarked three characteristic XRD peaks and several small XRD peaks at $2 \theta=2.22^{\circ}(0.02), 4.51^{\circ}(0.01), 6.76^{\circ}$ $(0.02), 11.32^{\circ}(0.02), 20.42^{\circ}(0.02)$, and $22.87^{\circ}(0.01)$
(Fig. $6 \mathrm{~A})$ and at $2 \theta=2.23^{\circ}(0.02), 4.50^{\circ}(0.01), 6.77^{\circ}(0.01)$, $11.33^{\circ}(0.01), 20.42^{\circ}(0.02)$, and $22.89^{\circ}(0.02)$ (Fig. 6B). The XRD pattern and $2 \theta$ values of diffraction peaks assigned to the developed MDP-Ca salt agreed with those 
(A)

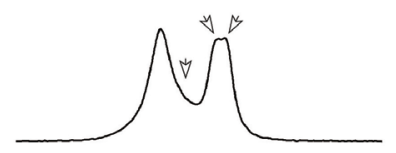

(B)

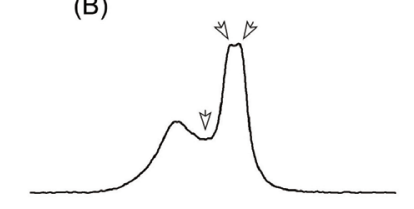

(C)

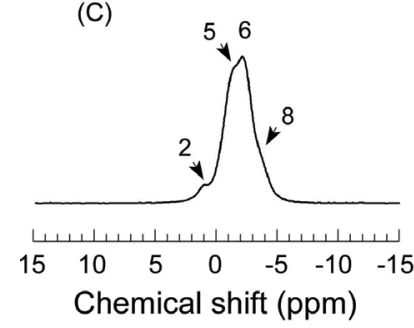

Fig. $7 \quad{ }^{31} \mathrm{P}$ NMR spectra of enamel and dentin reactants with experimental one-step adhesive and of the synthesized MDP-0.5 Ca salt, and the curve-fitting analysis results of the ${ }^{31} \mathrm{P}$ NMR spectra. Both enamel and dentin reactants were obtained after reacting with experimental one-step adhesive for $30 \mathrm{~s}$.

(A) ${ }^{31} \mathrm{P}$ NMR spectrum of enamel reactant; (B) ${ }^{31} \mathrm{P}$ NMR spectrum of dentin reactant; and (C) ${ }^{31} \mathrm{P}$ NMR spectrum of synthesized MDP-0.5 Ca salt. (a) Curve-fitting analysis result of ${ }^{31} \mathrm{P} \mathrm{NMR}$ spectrum of enamel reactant with adhesive; (b) Curve-fitting analysis result of ${ }^{31} \mathrm{P}$ NMR spectrum of dentin reactant with adhesive; and (c) Curvefitting analysis result of ${ }^{31} \mathrm{P}$ NMR spectrum of the synthesized MDP-0.5 Ca salt. The black line shows the original ${ }^{31} \mathrm{P}$ NMR spectrum of enamel reactant with adhesive, dentin reactant with adhesive or synthesized MDP-0.5 Ca salt, the purple line shows the simulated peak used for the curve-fitting of enamel or dentin, the sky-blue lines show the simulated peaks used for the curvefitting of each synthesized MDP-Ca salt, the pink line shows the simulated peak used for the curvefitting of DCPD with an amorphous phase, the red line shows the synthetic spectrum obtained by the curve-fitting analysis of enamel or dentin reactant with adhesive, and the green line is obtained after subtracting the synthetic spectrum from the original ${ }^{31} \mathrm{P}$ NMR spectrum of enamel or dentin reactant or synthesized MDP-0.5 Ca salt.

of the synthesized MDP-0.5 Ca salt (Fig. 6C). However, both enamel and dentin reactants did not show the XRD pattern assigned to DCPD with a crystalline phase.

${ }^{31} \mathrm{P}$ NMR analysis confirmed the XRD analysis results. A broad NMR peak showed two peaks at -1.41 and -2.17 ppm (Fig. 7A) or at -1.41 and $-2.17 \mathrm{ppm}$ (Fig. 7B), respectively assigned to the MDP-Ca salt in the NMR spectrum of enamel or dentin reactant with the adhesive. The spectral pattern of both broad NMR peaks was similar to that of synthesized MDP-0.5 Ca salt (Fig. 7C). However, we did not identify the existence of NMR peak "8" at the right side of the broad NMR peak in the NMR spectrum of enamel or dentin reactant, in contrast to that of synthesized MDP-0.5 Ca salt.

To gain an insight on the molecular species of MDP-Ca salts which were developed during the 30-s decalcification of enamel and dentin, and whether DCPD was indeed produced by decalcification, curvefitting analysis was performed on the ${ }^{31} \mathrm{P}$ NMR spectra of enamel and dentin reactants using simulated peaks. These simulated peaks were used for the curve-fitting analyses of three synthesized MDP-Ca salts, DCPD, and enamel or dentin (Tables 2 and 3). Here, we used simulated peak " $\gamma_{2}$ " to confirm the production of DCPD with an amorphous phase, since typical XRD pattern assigned to DCPD with a crystalline phase was not detected in the XRD patterns of both enamel and dentin reactants.

As shown in Fig. 7a, the ${ }^{31} \mathrm{P}$ NMR spectrum of enamel reactant with the adhesive was successfully curve-fitted by using simulated peak " $\alpha$ " for enamel apatite, simulated peak " $\gamma_{2}$ " for DCPD with an amorphous phase, and simulated peaks " 5 " and " 6 " for MDP-Ca salt. The ${ }^{31} \mathrm{P}$ NMR spectrum of dentin reactant (Fig. 7b) was completely curve-fitted by using simulated peak " $\beta$ " for dentin apatite, simulated peak " $\gamma_{2}$ " for DCPD with an amorphous phase, and simulated peaks " 3 ", " 5 " and " 6 " for MDP-Ca salt. The half width of simulated peak " $\gamma_{2}$ " for DCPD with an amorphous phase was $348.9 \mathrm{~Hz}$.

\section{DISCUSSION}

In this study, a curve-fitting analysis technique was used to determine the molecular species of MDP-Ca salts produced by 30 -s decalcification of enamel and dentin by experimental MDP-based one-step adhesive.

Based on the chemical shifts of eight simulated peaks used for the curve-fitting analyses of three synthesized MDP-Ca salts, simulated peaks " 1 ", “2", " 3 " and " 4 " were determined to be a phosphorus atom whose two hydroxy groups had ionized [R-O-P $\left.(\mathrm{O})\left(\mathrm{O}^{-}\right)_{2}\right]$. Simulated peaks " 5 ", " 6 ", " 7 " and " 8 " were determined to be a phosphorus atom in which one of the two hydroxy groups had ionized $\left[\mathrm{R}-\mathrm{O}-\mathrm{P}(\mathrm{O})(\mathrm{OH})\left(\mathrm{O}^{-}\right)\right]$. This is because the chemical shift of ${ }^{31} \mathrm{P}$ NMR peak is very sensitive to any change of the environment in which the phosphorus atom existed ${ }^{20)}$.

Based on the chemical shifts, intensities, intensity ratios, and intensity changes of eight simulated peaks, the molecular species which constituted the three synthesized MDP-Ca salts were determined and listed in Table 2. Simulated peaks " 2 " and "8" were respectively assigned to the phosphorus atom with two ionized hydroxy groups and the phosphorus atom with 
Table 4 Ratio of the molecular species constituted the three types of synthesized MDP-Ca salts

\begin{tabular}{|c|c|c|c|c|c|}
\hline & Molecular species & Peak & Intensity & Calibrated intensity ${ }^{\mathrm{a}}$ & Production Ratio \\
\hline \multirow{3}{*}{ MDP-0.5 Ca salt } & [ I ] & 5 & 0.451 & $0.451 / 1$ & 0.622 \\
\hline & [II ] & 6 & 0.513 & $0.513 / 2$ & 0.354 \\
\hline & {$[\mathrm{III}]$} & $2 \& 8$ & 0.035 & $0.035 / 2$ & 0.024 \\
\hline \multirow{6}{*}{ MDP-1 Ca salt } & [ I ] & 5 & 0.425 & $0.425 / 1$ & 0.565 \\
\hline & [II ] & 6 & 0.152 & $0.152 / 2$ & 0.101 \\
\hline & [III] & $2 \& 8$ & 0 & 0 & 0 \\
\hline & [IV] & 1 & 0.136 & $0.136 / 1$ & 0.181 \\
\hline & [V] & 3 & 0.116 & $0.116 / 2$ & 0.077 \\
\hline & [VI] & $4 \& 7$ & 0.171 & $0.171 / 3$ & 0.076 \\
\hline \multirow{6}{*}{ MDP-2 Ca salt } & [ I ] & 5 & 0.109 & $0.109 / 1$ & 0.205 \\
\hline & [II $]$ & 6 & 0.155 & $0.155 / 2$ & 0.145 \\
\hline & [III] & $2 \& 8$ & 0 & 0 & 0 \\
\hline & [IV] & 1 & 0.129 & $0.129 / 1$ & 0.243 \\
\hline & [V] & 3 & 0.094 & $0.094 / 2$ & 0.088 \\
\hline & [VI] & $4 \& 7$ & 0.513 & $0.513 / 3$ & 0.320 \\
\hline
\end{tabular}

a: Calibrated intensity was determined after dividing the intensity of each simulated peak by the number of phosphorus atom which has constituted the molecular species of MDP-Ca salt produced.

one ionized hydroxy group for MDP-Ca salt [III]. This was because the intensity ratio of simulated peak " 2 " to simulated peak "8" was approximately 1:1. Simulated peaks "4" and "7" were respectively assigned to the phosphorus atom with two ionized hydroxy groups and to two phosphorous atoms with one ionized hydroxy group for MDP-Ca salt [VI]. These assignments were possible because the intensity ratio of simulated peak "4" to simulated peak "7" was approximately 1:2.

Simulated peak "5", which had shifted at a lower field than simulated peak " 6 ", was assigned to the phosphorus atom with one ionized hydroxy group for MDP-Ca salt [I]. Simulated peak "6" was assigned to two phosphorus atoms with one ionized hydroxy group for MDP-Ca salt [II]. These assignments were due to a decrease in the intensity of simulated peak " 6 " with an increase in $\mathrm{CaCl}_{2} / \mathrm{MDP}$ ratio directly related to the production of MDP-Ca salt [V] and MDP-Ca salt [VI]. MDP-Ca salt [II] produced MDP-Ca salt [V] and MDP$\mathrm{Ca}$ salt $[\mathrm{VI}]$ after reaction with a calcium ion or $\mathrm{MDP}-\mathrm{Ca}$ salt [I].

Simulated peak "1" was assigned to the phosphorous atom with two ionized hydroxy groups for MDP-Ca salt [IV]. This was because simulated peak " 1 " shifted at the lowest field. Based on these analysis results, the production ratios of the molecular species of MDP-Ca salts, which constituted the series of three types of synthesized MDP-Ca salts (Table 4), were thus determined.

The production ratio of each molecular species of MDP-Ca salt changed with an increase in the molar ratio of $\mathrm{CaCl}_{2} / \mathrm{MDP}$. In light of these changes, the molecular species of MDP-Ca salt, which had provided asteriskmarked, black arrow-marked, or white arrow-marked three characteristic XRD peaks, were determined as follows. Asterisk-marked three characteristic XRD peaks $\left(2 \theta=2.24,4.51,6.76^{\circ}\right)$ were assigned to the layered structure of MDP-Ca salt [II]. This was because Fukegawa et $a l .{ }^{17)}$ reported that an MDP-Ca salt ([calcium bis(10methacryloyloxydecyl hydrogen phosphate), which was of the same molecular species as MDP-Ca salt [II], formed a layered structure and provided three characteristic XRD peaks at $2 \theta=2.24,4.56$, and $6.86^{\circ}$. Further, the observed decrease in the intensity of asterisk-marked three characteristic XRD peaks was directly related to a decrease in the production ratio of MDP-Ca salt [II].

The black arrow-marked three characteristic XRD peaks $\left(2 \theta=2.42,4.88,7.34^{\circ}\right)$ were assigned to the layered structure of MDP-Ca salt [V]. This was because the appearance of the black arrow-marked three characteristic XRD peaks agreed with the production of MDP-Ca salt [V]. The white arrow-marked three characteristic XRD peaks $\left(2 \theta=2.58,5.22,7.86^{\circ}\right)$ were assigned to the layered structure of MDP-Ca salt [VI]. This was because an increase in the production ratio of MDP-Ca salt [VI] corresponded to an increase in the intensity of the white arrow-marked three characteristic XRD peaks with an increase in the molar ratio of $\mathrm{CaCl}_{2} / \mathrm{MDP}$.

However, the intensities of the three types of characteristic XRD peaks did not relate to the production ratios of MDP-Ca salts [II], [V], and [VI]. MDP-Ca salt [V] provided a higher intensity of the three characteristic XRD peaks than MDP-Ca salt [II], although the production ratio of MDP-Ca salt [V] was less than those of MDP-Ca salts [II] and [VI]. It was probable that the crystallinity of the layered structure that the MDP-Ca salt had formed differed among the three types of MDP-Ca salts [II], [V], and [VI]. 
Table 5 Peak intensity ratio of unreacted enamel or dentin, DCPD and MDP-Ca salts and Production ratios of molecular species of MDP-Ca salts [I], [II], and [V]

\begin{tabular}{lcccc}
\hline & Peak & Intensity ratio & $\begin{array}{c}\text { Molecular species } \\
\text { of MDP-Ca salt }\end{array}$ & $\begin{array}{c}\text { Production ratio of each } \\
\text { molecular species }\end{array}$ \\
\hline Unreacted Enamel & $\alpha$ & $0.475(0.014)$ & - & - \\
Produced DCPD & $\gamma_{2}$ & $0.121(0.007)$ & {$[\mathrm{I}]$} & $0.689(0.001)$ \\
Produced MDP-Ca salt & 5 & $0.212(0.011)$ & - & $0.311(0.001)$ \\
\hline Unreacted Dentin & 6 & $0.192(0.010)$ & - & - \\
Produced DCPD & $\beta$ & $0.355(0.004)$ & {$[\mathrm{II}]$} & - \\
& & $0.061(0.005)$ & $0.077(0.001)$ \\
Produced MDP-Ca salt & $\gamma_{2}$ & $0.067(0.000)$ & $0.649(0.009)$ \\
& 3 & $0.280(0.007)$ & $0.274(0.008)$ \\
\hline
\end{tabular}

(SD)

Curve-fitting analyses clearly showed that enamel and dentin developed several types of MDP-Ca salts and DCPD with an amorphous phase during the 30-s decalcification (Table 5). This was because simulated peaks " 5 ", "6" and " $\gamma_{2}$ " respectively evidenced the production of MDP-Ca salt [I] (a mono-calcium salt of MDP monomer), MDP-Ca salt [II] (a mono-calcium salt of MDP dimer), and DCPD with an amorphous phase. Dentin also produced MDP-Ca salt [V], a dicalcium of MDP dimer alongside the above two types of MDP-Ca salts. This was due to dentin eluting greater amounts of calcium and phosphate ions into the experimental adhesive through the decalcification process than enamel, since the decalcification rate is strongly influenced by the crystal structure and size of hydroxyapatite —which constitutes enamel and dentin ${ }^{16)}$. The predominant molecular species of MDPCa salt produced by decalcification of enamel or dentin was a mono-calcium salt of MDP monomer. In contrast, the production ratio of the mono-calcium salt of MDP dimer which provided three characteristic XRD peaks was less than $31 \%$.

NMR analysis is a very powerful technique to investigate the decalcification mechanisms of enamel and dentin by one-step MDP-based adhesive. We were able to detect ${ }^{31} \mathrm{P}$ NMR peaks assigned to MDP-Ca salts and DCPD with an amorphous phase produced by decalcification in the same way as those with a crystalline phase, in contrast to XRD analysis technique.

\section{CONCLUSIONS}

Curve-fitting analyses of the ${ }^{31} \mathrm{P}$ NMR spectra of both enamel and dentin reactants with the adhesive demonstrated that enamel and dentin produced several types of MDP-Ca salts and DCPD with an amorphous phase. The molecular species of MDP-Ca salts developed by the 30-s decalcification of enamel and dentin were mono-calcium salts of MDP monomer and MDP dimer.
Dentin also produced a di-calcium of MDP dimer.

\section{ACKNOWLEDGMENTS}

This work was supported by a grant-in-aid for Developmental Scientific Research from the Ministry of Education, Culture, Sports, Science and Technology in Japan (No. 25462969) and a grant from the Research Institute of Oral Science, Nihon University School of Dentistry at Matsudo.

The XRD experiments were performed at the Laboratory for Electron Beam Research and Application (LEBRA), Nihon University. We would like to express our sincere thanks to Professor Moriyasu Wada and Professor Toshiro Sakae.

\section{REFERENCES}

1) Pashley EL, Agee KA, Pashley DH, Tay FR. Effect of one versus two applications of an unfilled, all-in-one adhesive on dentine bonding. J Dent 2002; 30: 83-90.

2) Van Meerbeek B, De Munck J, Yoshida Y, Inoue S, Vargas M, Vijay P, Van Landuyt K, Lambrechts P, Vanherle G. Buonocore memorial lecture. Adhesion to enamel and dentin: current status and future challenges. Oper Dent 2003; 28: 215-235.

3) Koshiro K, Sidhu SK, Inoue S, Ikeda T, Sano H. New concept of resin-dentin interfacial adhesion: the nanointeraction zone. J Biomed Mater Res B Appl Biomater 2006; 77: 401-408.

4) Nishiyama N, Suzuki K, Takahashi K, Nemoto K. The pKa effects of the carboxylic acid in N-methacryloyl-omega-amino acid on the demineralization and bond strengths to the teeth. Biomaterials 2004; 25: 5441-5447.

5) Nishiyama N, Fujita K, Ikemi T, Maeda T, Suzuki K, Nemoto K. Efficacy of varying the NMEP concentrations in the NMGly-NMEP self-etching primer on the resin-tooth bonding. Biomaterials 2005; 26: 2653-2661.

6) Nishiyama N, Aida M, Fujita K, Suzuki K, Tay FR, Pashley DH, Nemoto K. NMR study on the adhesion efficacy of experimental phosphonic acid monomers. Dent Mater J 2007; 26: 382-387.

7) Fujita K, Nishiyama N. ${ }^{13} \mathrm{C}$ NMR analysis of the etching 
efficacy of acidic monomers in self-etching primers. J Dent 2006; 34: 123-133.

8) Fujita K, Ma S, Aida M, Maeda T, Ikemi T, Hirata M, Nishiyama N. Effect of reacted acidic monomer with calcium on bonding performance. J Dent Res 2011; 90: 607-612.

9) Iwai H, Nishiyama N. Effect of calcium salt of functional monomer on bonding performance. J Dent Res 2012; 91: 1043-1048,

10) Li N, Nikaido T, Takagaki T, Sadr A, Makishi P, Chen J, Tagami J. The role of functional monomers in bonding to enamel: Acid-base resistant zone and bonding performance. J Dent 2010; 38: 722-730.

11) Nikaido T, Ichikawa C, Li N, Takagaki T, Sadr A, Yoshida Y, Suzuki K, Tagami J. Effect of functional monomers in allin-one adhesive systems on formation of enamel/dentin acidbase resistant zone. Dent Mater J 2011; 30: 576-582.

12) Yoshida Y, Van Meerbeek B, Nakayama Y, Snauwaert J, Hellemans L, Lambrechts P, Vanherle G, Wakasa K. Evidence of chemical bonding at biomaterial-hard tissue interfaces. J Dent Res 2000; 79: 709-714.

13) Yoshida Y, Van Meerbeek B, Nakayama Y, Yoshioka M, Snauwaert J, Abe Y, Lambrechts P, Vanherle G, Okazaki M. Adhesion to and decalcification of hydroxyapatite by carboxylic acids. J Dent Res 2001; 80: 1565-1569.

14) Yoshida Y, Nagakane K, Fukuda R, Nakayama Y, Okazaki M, Shintani H, Inoue S, Tagawa Y, Suzuki K, De Munck J, Van
Meerbeek B. Comparative study on adhesive performance of functional monomers. J Dent Res 2004; 83: 454-458.

15) Yoshihara K, Yoshida Y, Hayakawa S, Nagaoka N, Irie M, Ogawa T, Van Landuyt KL, Osaka A, Suzuki K, Minagi S, Van Meerbeek B. Nanolayering of phosphoric acid ester monomer on enamel and dentin. Acta Biomater 2011; 7: 3187 3195.

16) Yoshida Y, Yoshihara K, Nagaoka N, Hanabusa M, Matsumoto T, Momoi Y. X-ray diffraction analysis of three-dimensional self-reinforcing monomer and its chemical interaction with tooth and hydroxyapatite. Dent Mater J 2012; 31: 697-702.

17) Fukegawa D, Hayakawa S, Yoshida Y, Suzuki K, Osaka A, Van Meerbeek B. Chemical interaction of phosphoric acid ester with hydroxyapatite. J Dent Res 2006; 85: 941-944.

18) Yoshihara K, Yoshida Y, Nagaoka N, Fukegawa D, Hayakawa S, Mine A, Nakamura M, Minagi S, Osaka A, Suzuki K, Van Meerbeek B. Nano-controlled molecular interaction at adhesive interfaces for hard tissue reconstruction. Acta Biomater 2010; 6: 3573-3582.

19) Takahashi H. Effect of calcium salt of 10-methacryloxydecyl dihydrogen phosphate produced on the bond durability of one-step self-etch adhesive. Dent Mater J 2014; 33: 394-401.

20) Fu B, Sun X, Qian W, Shen Y, Chen R, Hannig M. Evidence of chemical bonding to hydroxyapatite by phosphoric acid esters. Biomaterials 2005; 26: 5104-5110. 